Revista de Matemática: Teoría y Aplicaciones 2005 12(1 \& 2) : 73-88

CIMPA - UCR - CCSS ISSN: 1409-2433

\title{
INDENTIFICACIÓN DE FASES DE LA DIABETES ESPONTÁNEA DE UN BIOMODELO MURINO MEDIANTE ANÁLISIS MULTIDIMENSIONAL DE DATOS
}

\author{
Nora Moscoloni* Silvana M. Montenegro ${ }^{\dagger}$ Hugo D. Navone ${ }^{\ddagger}$ \\ Juan Carlos Picena ${ }^{\S}$ Stella M. Martínez M. Cristina Tarrés"l
}

Recibido/Received: 17 Feb 2004

\begin{abstract}
Resumen
Los biomodelos utilizados para el estudio de la diabetes permiten evaluar factores genéticos y ambientales. Nuestro propósito fue caracterizar individuos de la línea de ratas genéticamente diabéticas eSS utilizando, mediante análisis multivariado, los valores de la curva de tolerancia glúcida y de glucosuria, junto con otras características fisiológicas y ambientales totalizando 9 variables. Se asignaron valores faltantes de glucosuria mediante un clasificador neuronal. Para la caracterización de los individuos se aplicó el método de componentes principales y al efectuar la descripción de la estructura de los datos mediante representación gráfica en ejes factoriales, el primer eje separó los individuos según las glucemias, edad y peso y el segundo opuso la biomasa en edades tempranas con el tamaño de camada. El análisis en clusters definió una partición en 5 clases. Al relacionar los resultados con la clasificación clínica fue posible tipificar a los machos $e S S$ desde los más jóvenes con menor peso, aglucosúricos, con glucemia de ayuno normal pero con alteración de la tolerancia a la glucosa hasta los diabéticos, de mayor peso y edad y glucosúricos, posibilitándose así la identificación de fases en la progresión del síndrome.
\end{abstract}

* Programa Interdisciplinario de Análisis de Datos (PIAD), IRICE (CONICET) y Universidad Nacional de Rosario (UNR). Maipú 1065, of.203, (2000) Rosario, Argentina. Tel. +(54)(341)4201275, Fax +(54)(341)4201259. E-Mail: nmoscolo@sede.unr.edu.ar, piad@sede.unr.edu.ar.

${ }^{\dagger}$ Facultad de Ciencias Médicas, Consejo de Investigaciones de la Universidad Nacional de Rosario (UNR). Santa Fe 3100, (2000) Rosario, Argentina. E-Mail: smontene@unr.edu.ar.

${ }^{\ddagger}$ Facultad de Ciencias Exactas, Ingeniería y Agrimensura (UNR) e Instituto de Física Rosario (CONICET), Bv. 27 de Febrero 210 bis, (2000) Rosario, Argentina. E-Mail: hnavone@ifir.edu.ar.

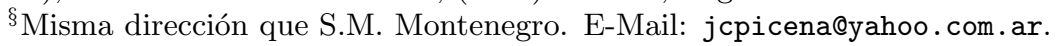

"Misma dirección que S.M. Montenegro. E-Mail: smartinez@telnet.com.ar.

"Misma dirección que S.M. Montenegro. E-Mail: mctarres@rsinternet.com.ar. 
Palabras clave: análisis multidimensional de datos, diabetes, biomodelos, redes neuronales artificiales.

\begin{abstract}
Biomodels used in the study of diabetes allow to evaluate genetic and environmental factors. Our aim was to characterize individuals of eSS, a genetically diabetic line of rats. We applied multivariate analysis, using the values obtained during the performance of oral glucose tolerance tests, presence of glucosuria, together with other physiological and environmental characteristics totalling 9 variables. Previously, an assignation of missing values of glucosuria was carried out through an artificial neural network classifier. To characterize individuals, principal componentes analysis was carried out. On describing data structure in a graphical representation of factorial coordinates, the first axe separated individuals according to glycemias, age and weight and the second opposed biomass in early ages to litter size. The cluster analysis defined a typology based on five classes. When these results were correlated with clinical classification, it was possible to separate eSS males from the youngest rats with low body weight, aglucosuric, with normal fasting glycemia but impaired glucose tolerance, up to diabetic individuals, older, with higher biomass and glucosuric. This methodology allows to identify stages in the progression of the diabetic syndrome.
\end{abstract}

Keywords: multivariate data analysis, diabetes, biomodels, artificial neural network.

Mathematics Subject Classification: 92B05, 95B10, 95B15, 95B20.

\title{
1 Introducción
}

La diabetes mellitus constituye un grupo de enfermedades etiológica y clínicamente heterogéneo, caracterizado por hiperglucemia crónica y otras anormalidades metabólicas, debidas a una deficiencia en la acción de la insulina (Kuzuya et al. 2002). Su prevalencia aumenta año tras año y, en muchos países, alcanza valores superiores al $5 \%$ de la población total (Ramos et al. 2000, Roselló-Araya 2003).

El Comité de Expertos en Diabetes (American Diabetes Association 2004) propuso mantener la diferenciación en diabetes "tipo 1" y "tipo 2". El tipo 1 incluye los casos atribuíbles principalmente a procesos autoinmunes y el tipo 2 abarca la mayoría de las formas prevalentes de diabetes, que resultan de resistencia a la insulina, con defectos en la secreción o acción de dicha hormona. Tiene gran variabilidad en su expresión, importantes componentes genéticos y se ve afectada significativamente por factores ambientales (Costa et al 2002, Roselló-Araya 2003).

Han sido descriptas numerosas líneas de roedores de laboratorio que constituyen biomodelos de diabetes espontánea (Shafrir 1996). Su utilización ha colaborado de modo significativo al conocimiento actual de variados aspectos de la enfermedad (Buschard 2001), ya que imitan la fisiopatología del síndrome diabético en el ser humano (van Zutphen 1999). El Comité de Expertos en diabetes de la OMS ha recomendado tanto su empleo como su desarrollo (Informe de un Grupo de Estudio de la OMS 1985).

La línea de ratas $e S S$, desarrollada, criada y estudiada en la Cátedra de Biología de la Facultad de Ciencias Médicas de Rosario, deriva de la cepa IIM de ratas albinas 
(Supplement IV of the International Survey on the Supply, Quality and Use of Laboratory Animals 1964). Fue designada $e S S$ (Tarrés et al. 1981) e inscripta internacionalmente bajo la denominación completa $I I M e / F m$ eSS (Calderari et al. 1991). Los animales $e S S$ no son obesos ni exhiben un importante acortamiento de su vida (Tarrés et al. 1992, Martínez et al. 1993), hecho que contribuye para que esta línea haya sido reconocida como modelo biológico para el estudio de la diabetes tipo 2 y sus complicaciones crónicas (Tarrés et al. 1992, Martínez et al. 1993, Gagliardino 2000, Picena et al. 2002). Su elevada endocría teórica, mayor a 0.92 en el año 1980 (Tarrés et al. 1981) fue corroborada mediante la aceptación en un $100 \%$ de transplantes singeneicos de piel de la cola en machos adultos (Calderari et al. 1995).

La genética sostiene que la estructura y función de un organismo dependen de factores genéticos y ambientales (Solari 1999). Así es que, para algunos genotipos entre los que se halla el diabético, dicho plan puede ser altamente variable y producir fenotipos sensibles a cambios en el ambiente (Strickberger 1988, Pereira et al. 1995, Uusitupa 1996).

El objetivo del presente trabajo consistió en clasificar fenotípicamente animales de la línea de ratas diabéticas eSS para analizar efectos derivados de factores ambientales internos (edad, peso) y externos (estacionalidad, relaciones nutricionales maternas) en una población cuyo elevado coeficiente de endocría garantiza, al menos teóricamente, uniformidad genotípica.

\section{Material y métodos}

Se utilizaron 484 machos $e S S$ en los que se registraron las siguientes variables:

- G0: glucemia de ayuno $(\mathrm{mg} / \mathrm{dl})$,

- glucemias a los $30(G 30), 60(G 60)$ y a los $120(G 120)$ minutos de una sobrecarga oral con glucosa $(\mathrm{mg} / \mathrm{dl})$,

- presencia $(G+)$ o ausencia $(G-)$ de glucosuria, determinada en la orina emitida por micción espontánea durante la ejecución de la curva de tolerancia glúcida,

- edad (en días) y peso (g) en el momento de efectuar las determinaciones bioquímicas,

- mes del año en que se realizó la curva de tolerancia glúcida,

- peso (g) al nacimiento,

- tamaño de la camada (número de crías nacidas en el mismo momento y que comparten ambos progenitores),

- mes de nacimiento,

- peso (g) al destete (21 días).

El análisis estadístico comprendió tres etapas: 


\subsection{Preprocesamiento de datos usando redes neuronales artificiales}

La recolección de la orina emitida por micción espontánea para determinar la glucosuria tiene el problema de arrojar una gran cantidad de datos faltantes — en nuestro caso 220cifra que corresponde al $45 \%$ del total de los casos estudiados. Dado que la intensidad del síndrome diabético puede expresarse operacionalmente por los niveles de glucemia y por la presencia o no de glucosuria y considerando, por lo tanto, la importancia de contar con el valor de esta variable para identificar diferentes tipos de individuos, se procedió a estimar los valores desconocidos en base al conjunto de animales en donde había podido ser registrada.

La elección del método para la asignación de los valores faltantes se realizó de acuerdo a los siguientes criterios:

- independencia respecto del análisis que posteriormente se llevó a cabo para la determinación de la tipología de individuos ,

- alta flexibilidad de la técnica a los efectos de obtener un modelo predictivo con suficiente capacidad de generalización.

Puesto que las redes neuronales artificiales satisfacen ambos criterios, se diseñó un clasificador neuronal para la asignación de los datos faltantes.

En coincidencia con el número de variables que describen a un individuo, se fijó en 12 el número de unidades de la capa de entrada del clasificador, en 3 el número de unidades de la capa intermedia y en 2 el número de unidades de la capa de salida, dado que el número de modalidades de la variable nominal $G$ es $2(G+$ y $G-)$.

Se utilizó el método softmax para representar las respuestas del clasificador y la técnica de retropropagación de errores para el ajuste de los parámetros del modelo (Duda et al. 2001). Se empleó la metodología de validación cruzada múltiple para obtener diversos clasificadores neuronales y se estimó la capacidad de generalización de cada uno de ellos usando un conjunto de datos o test totalmente independiente al utilizado en los procesos de ajuste.

La asignación final de los valores faltantes de glucosuria se realizó mediante el agregado de los distintos clasificadores obtenidos durante el proceso de validación múltiple, tomando como clase adjudicada a la que obtuvo la máxima frecuencia de selección (Dietterich 2000).

\subsection{Análisis de Componentes Principales}

El enfoque multivariado incluye una serie de técnicas estadísticas que permiten considerar de manera simultánea un conjunto de variables medidas en un conjunto de individuos (Carrasco y Hernán 1993), entre ellas el análisis de componentes principales es una técnica factorial que se utiliza para resumir un conjunto de variables continuas en un número pequeño de variables sintéticas. (Escofier y Pagès 1992, Aluja y Morineau 1999).

Este método transforma el conjunto original en uno más pequeño de combinaciones lineales de las variables de origen, las cuales representan la mayor parte de la variancia del conjunto original. El objetivo es determinar factores o componentes principales que expliquen la mayor variación posible de los datos con la menor cantidad de dichos factores. 
El enfoque de la escuela francesa del análisis de datos posibilita la elección de variables activas e ilustrativas o suplementarias. Las primeras son las que se incluyen en los cálculos de diagonalización de la matriz mientras que las segundas se proyectan a posteriori en el espacio factorial construido con las variables activas.

Como activas fueron consideradas las variables continuas $G 0, G 30, G 60, G 120$, edad y peso en el momento de efectuar las determinaciones bioquímicas, tamaño de la camada, peso al nacimiento y peso al destete, y como ilustrativas las nominales mes del año en que se realizó la curva de tolerancia glúcida, mes de nacimiento y glucosuria.

\subsection{Construcción de una tipología de individuos}

Este método se refiere a la formación de clases o clusters con características semejantes permitiendo la observación de las variables que se encuentran asociadas (Lebart, Morineau et al. 1995), teniendo como objetivo reagrupar los individuos en un número restringido de clases homogéneas y bien separadas.

Para la construcción de la tipología se tuvo en cuenta las coordenadas de los mismos en los 2 primeros ejes factoriales considerando este subespacio como el más significativo, dada la relevancia del efecto tamaño para los objetivos de este trabajo y se aplicó la estrategia de clasificación mixta de extendida aplicación en muestras grandes (Moscoloni 1992).

El algoritmo de clasificación mixta procede en tres fases. En primer lugar el conjunto de los elementos a clasificar es sometido a una partición inicial a través del método de centros móviles, que puede ser considerado como un caso particular de las técnicas conocidas con el nombre de nubes dinámicas (Diday 1971). Mediante este método se obtuvieron varias decenas de grupos homogéneos. Como diferentes particiones pueden dar lugar a diferentes agrupamientos, se realizó un cruce de las particiones construidas obteniendo así los llamados grupos estables o formas fuertes. Estos grupos de individuos, que aparecieron siempre en las mismas clases, fueron los elementos de base de la etapa siguiente.

La segunda etapa consistió en efectuar una clasificación ascendente jerárquica donde los elementos terminales del árbol fueron las clases de la partición anterior. El árbol fue construido según el criterio de Ward.

La partición final fue definida por corte del árbol correspondiente y la homogeneidad de las clases obtenidas fue optimizada por reasignaciones realizadas nuevamente con el procedimiento de agregación alrededor de centros móviles.

Finalmente, se realizó una descripción del contenido de las clases a partir de las variables activas e ilustrativas. El procedimiento permite la interpretación de las clases formadas en términos de las variables originales, utilizándose un criterio para jerarquizar las modalidades más características basado en el cálculo de valores tests en el entorno de una distribución Normal $(0,1)$. Para ello se realizó una comparación de medias, respondiendo a la idea de considerar una variable continua como más característica de un grupo si su promedio es significativamente diferente del general, que corresponde al del conjunto total de individuos.

El procesamiento estadístico se efectuó con el software SPAD (1999). 


\section{Resultados}

La variable ilustrativa glucosuria pudo evaluarse en 264 animales siendo positiva en el $62 \%$ de los casos. Los clasificadores neuronales obtenidos a partir de la implementación del proceso de validación múltiple, permitieron clasificar correctamente a los datos del conjunto separado como test con una performance promedio del $86 \%$ y con un desvío estándar del 4\%. El agregado de clasificadores se aplicó al conjunto de individuos en el que la variable glucosuria no estaba determinada, resultando así un valor asignado para este dato faltante.

La descripción simultánea de la estructura de los datos efectuada a través del análisis de componentes principales, puede visualizarse mediante una representación gráfica en ejes factoriales. Los dos primeros, acumularon una inercia de 56.73\%, los intervalos laplacianos de Anderson (1963) justificaron significativamente la desigualdad de estos dos valores propios, pudiéndose aplicar además la regla del "codo" de Cattell (1966).

Sobre un plano dividido en cuadrantes, se ubican las variables activas de acuerdo al valor de las correlaciones obtenidas, observando en la Figura 1 que, con referencia al primer factor, todas las variables están en el mismo lado respecto del origen de coordenadas. Tal disposición evidencia el "efecto tamaño" característico de los datos biométricos, traduciendo el hecho que la mayoría están positivamente correlacionadas entre sí por lo que el primer eje factorial separa los individuos según los dosajes de $G 0, G 30, G 60$ y G120, y edad y peso en el momento de realizar la curva de tolerancia glúcida. El hecho de que la variable edad se encuentre incluida en este efecto es particularmente relevante para los objetivos de este trabajo, es decir para la determinación de fases de la enfermedad. El segundo eje factorial opone los valores del peso al nacimiento y a los 21 días de los correspondientes al tamaño de la camada dando cuenta de la falta de relación entre estas últimas variables con las primeras y poniendo de manifiesto la relación inversa entre esas variables ya señalada por otros autores (Delemarre-van de Waal et al. 2002, Poulos et al. 2001).

Se realizó luego un análisis en clusters para agrupar los individuos según sus afinidades en las variables estudiadas, aplicando las técnicas de clasificación sobre las coordenadas de los individuos en los ejes factoriales. A partir del árbol jerárquico o dendrograma construido mediante el algoritmo ascendente, según el cual las clases se van reagrupando progresivamente, se definió una partición en 5 clases. La Figura 2 representa al árbol obtenido o dendrograma.

Se proyectaron los individuos en el gráfico factorial, identificados por su número de clase, y se obtuvieron los agrupamientos que se muestran en la Figura 3. En ella se observa también la ubicación de las modalidades "positiva y negativa" de la variable ilustrativa glucosuria, correspondiendo destacar la proximidad existente entre los valores originales y adjudicados.

La configuración de los clusters a través de las variables activas y de la ilustrativa glucosuria así como las comparaciones entre los promedios o porcentajes de las clases respecto del general se observan en la Tabla 1. Las variables ilustrativas mes de nacimiento y mes del año en que se realizó la curva de tolerancia glúcida no efectuaron aportes significativos dado que la distribución de sus modalidades resultó proporcional y, por 


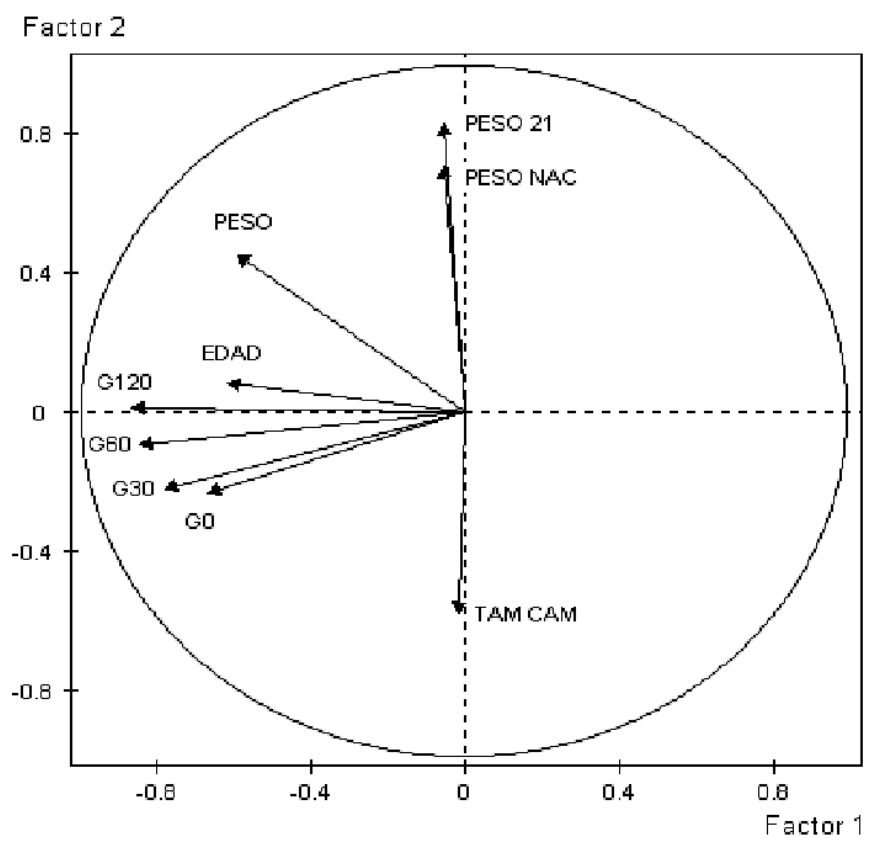

Figura 1: Representación gráfica de las variables activas en los ejes factoriales.

lo tanto, no resultó posible determinar tendencia alguna de agrupamiento en las clases referidas.

En relación con las variables activas, de la Tabla 1 se desprende que:

- las glucemias, la edady el peso se ubican, en términos generales, en orden decreciente; esto es, mayores valores en las primeras clases y menores en las últimas.

Respecto de la variable ilustrativa glucosuria puede visualizarse que:

- la modalidad glucosuria positiva (original y adjudicada) se ubica en orden decreciente; vale decir, mayores porcentajes en las primeras clases y menores en las últimas, coincidiendo en general con el ordenamiento de las glucemias;

- la modalidad glucosuria negativa tanto original como adjudicada lo hace en orden creciente desde la clase 3 a la clase 5 , no registrándose valores de esta categoría en las clases 1 y 2 .

\section{Discusión}

En el presente estudio, el estado diabético de las ratas $e S S$ fue evaluado mediante los valores de la curva de tolerancia a la glucosa, considerada irreemplazable para la identificación de pacientes con altos valores de glucemia postprandial (Herdzik et al. 2002). 


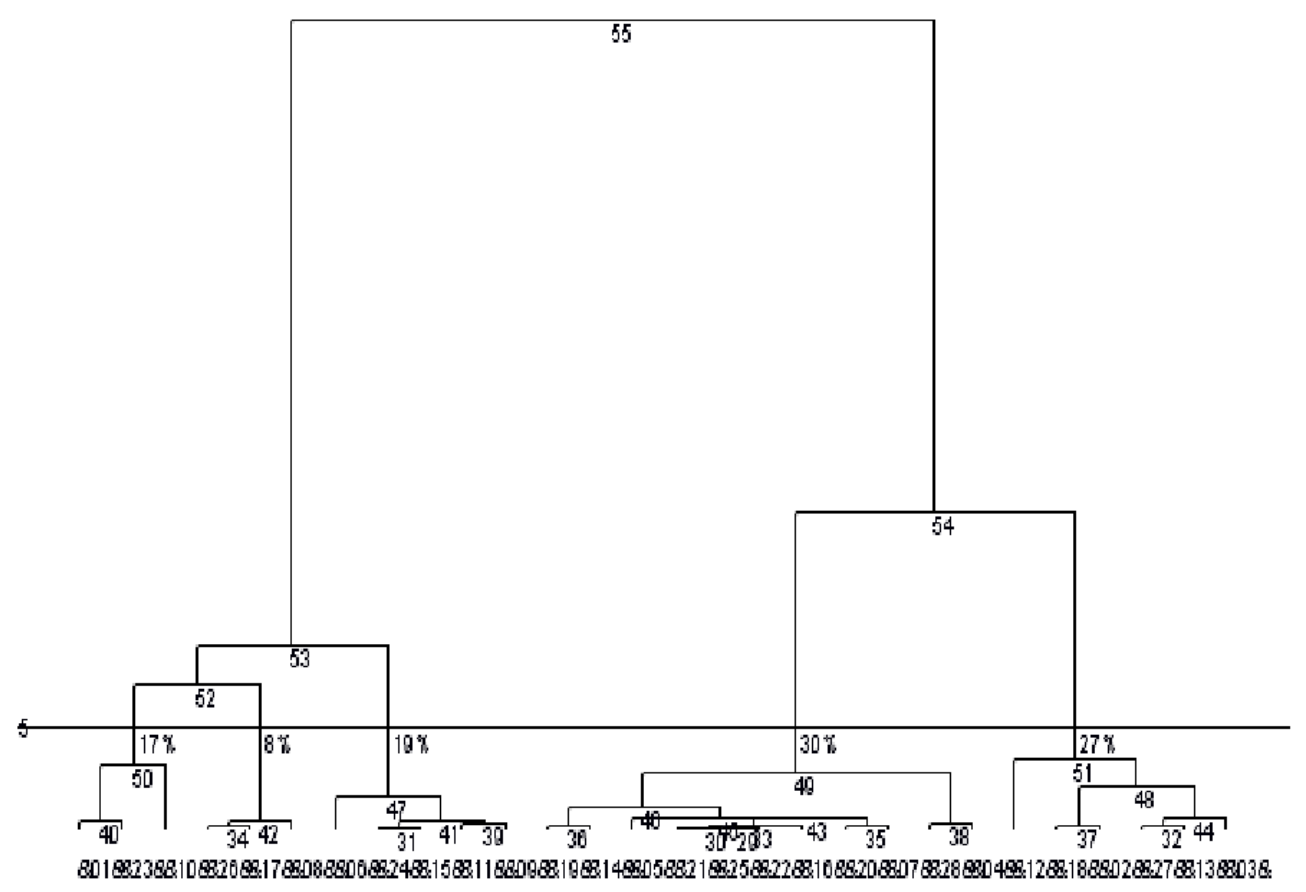

Figura 2: Representación gráfica del árbol o dendrograma.

Con referencia a ella, el Comité de Expertos para el Diagnóstico y Clasificación de la Diabetes Mellitus de la American Diabetes Association (2004), revisó y propuso la siguiente clasificación de diabetes y otras categorías de regulación de la glucosa, correspondiendo $G 0$ y $G 120$ a las glucemias de ayuno y a los 120 minutos de la sobrecarga glúcida: $\mathrm{Si}$

- $G 0<110 \mathrm{mg} / \mathrm{dl}:$ glucemia de ayuno normal,

- $110 \leq G 0<126 \mathrm{mg} / \mathrm{dl}:$ glucemia de ayuno alterada,

- $G 0 \geq 126 \mathrm{mg} / \mathrm{dl}$ : diagnóstico provisional de diabetes,

- $G 120<140 \mathrm{mg} / \mathrm{dl}$ : tolerancia normal a la glucosa,

- $140 \mathrm{mg} / \mathrm{dl} \leq G 120<200 \mathrm{mg} / \mathrm{dl}:$ tolerancia a la glucosa alterada,

- $G 120 \geq 200 \mathrm{mg} / \mathrm{dl}$ : diagnóstico provisional de diabetes.

En esta clasificación los términos tolerancia a la glucosa alterada y glucemia de ayuno alterada se refieren a estados metabólicos intermedios entre una normal homeostasis de la glucosa y la diabetes.

Los valores de la glucemia a los 30 y 60 minutos de la sobrecarga con glucosa también han sido señalados como factores con poder diagnóstico. Traversa y Ruiz (1994) postulan 


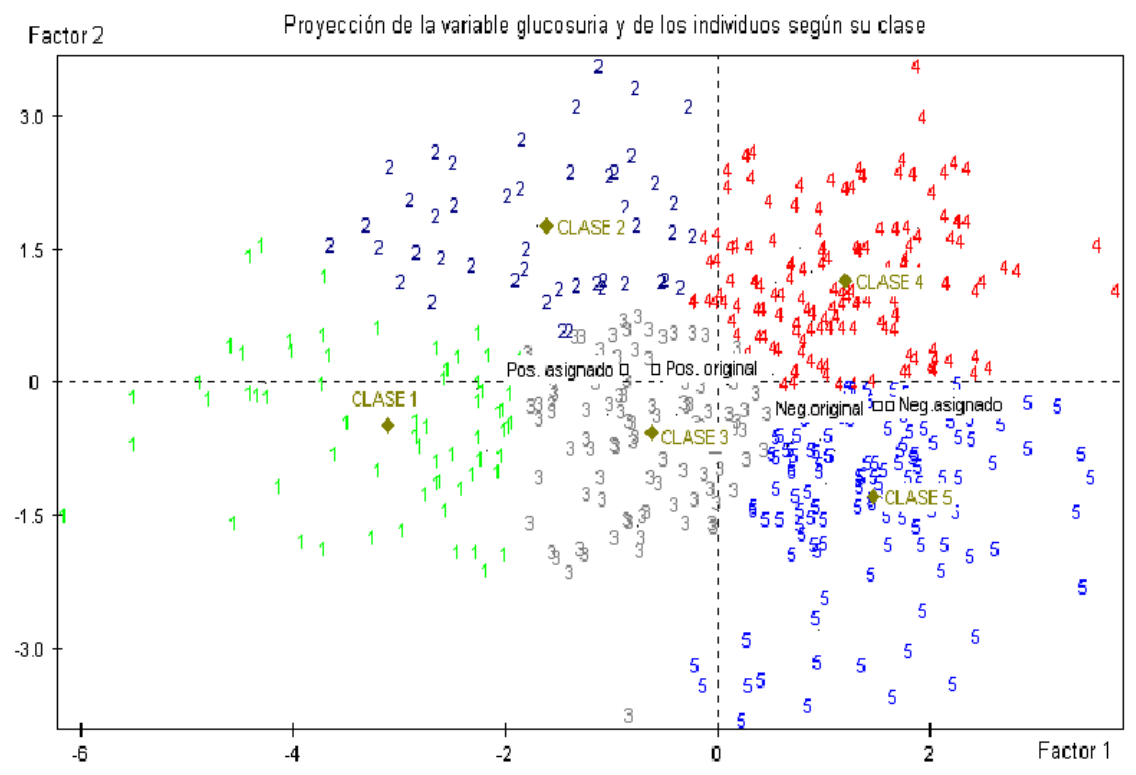

Figura 3: Proyección de los individuos según su clase y de las modalidades de la variable glucosuria.

como indicadores de diabetes ambos dosajes mayores a $200 \mathrm{mg} / \mathrm{dl}$, situación que en nuestra tipificación se presenta en todas las clases, con un ordenamiento creciente desde la 1 hasta la 5 acompañando a los valores de G0 y G120.

Con respecto a la variable glucosuria, la metodología de estimación de modelos predictivos mediante redes neuronales artificiales, ha sido aplicada con éxito en una amplia gama de problemas (Duda et al. 2001). En nuestro caso resultó pertinente para la adjudicación de datos faltantes, dada la importancia de la variable glucosuria en los estudios de diabetes, tanto para su diagnóstico como para su control periódico, evolución y tratamiento (Kokko 1996). En los resultados obtenidos, puede interpretarse que el elevado porcentaje de las glucosurias positivas adjudicadas en la clase 1 podría deberse a la estrecha vinculación entre el monto de la hiperglucemia y la presencia de glucosuria, siendo importante señalar que no se encuentran en esta clase individuos con glucosurias negativas. Con este mismo razonamiento puede explicarse, en la clase 5, la menor adjudicación de glucosurias negativas respecto de las observadas.

La edad es una variable que denota un ambiente interno determinado, siendo un indicador de los cambios fisiológicos que se producen en un individuo con el paso del tiempo. Ha mostrado su influencia agravando las perturbaciones metabólicas en pacientes diabéticos (Tsukui y Kobayashi 1995), en el Macacus rhesus (Hansen y Bodkin 1986), en 


\begin{tabular}{|c|c|c|c|c|c|c|c|c|c|c|c|}
\hline \multirow[t]{2}{*}{ Variables } & \multirow{2}{*}{$\begin{array}{c}\text { General } \\
\bar{x} \pm \sigma\end{array}$} & \multicolumn{2}{|c|}{$\begin{array}{l}\text { Clase } 1 \\
n=66\end{array}$} & \multicolumn{2}{|c|}{$\begin{array}{l}\text { Clase 2 } \\
n=46\end{array}$} & \multicolumn{2}{|c|}{$\begin{array}{c}\text { Clase } 3 \\
n=104\end{array}$} & \multicolumn{2}{|c|}{$\begin{array}{c}\text { Clase } 4 \\
n=144\end{array}$} & \multicolumn{2}{|c|}{$\begin{array}{c}\text { Clase } 5 \\
n=120\end{array}$} \\
\hline & & $\bar{x} \pm \sigma$ & $p$ & $\bar{x} \pm \sigma$ & $p$ & $\bar{x} \pm \sigma$ & $p$ & $\bar{x} \pm \sigma$ & $p$ & $\bar{x} \pm \sigma$ & $p$ \\
\hline$G 0$ & $104 \pm 31$ & $147 \pm 31$ & 0.000 & $114 \pm 26$ & 0.013 & $107 \pm 26$ & 0.165 & $86 \pm 18$ & 0.000 & $98 \pm 26$ & 0.003 \\
\hline G30 & $269 \pm 70$ & $369 \pm 65$ & 0.000 & $290 \pm 62$ & 0.014 & $294 \pm 44$ & 0.000 & $222 \pm 43$ & 0.000 & $238 \pm 47$ & 0.000 \\
\hline$G 60$ & $335 \pm 90$ & $466 \pm 62$ & 0.000 & $389 \pm 55$ & 0.000 & $367 \pm 55$ & 0.000 & $283 \pm 63$ & 0.000 & $276 \pm 60$ & 0.000 \\
\hline$G 120$ & $270 \pm 100$ & $412 \pm 78$ & 0.000 & $353 \pm 67$ & 0.000 & $311 \pm 61$ & 0.000 & $212 \pm 57$ & 0.000 & $194 \pm 51$ & 0.000 \\
\hline Peso & $336 \pm 51$ & $376 \pm 36$ & 0.000 & $396 \pm 44$ & 0.000 & $342 \pm 35$ & 0.068 & $331 \pm 32$ & 0. 083 & $291 \pm 49$ & 0.000 \\
\hline Edad & $281 \pm 124$ & $418 \pm 106$ & 0.000 & $374 \pm 119$ & 0.000 & $301 \pm 119$ & 0.027 & $230 \pm 83$ & 0.000 & $212 \pm 85$ & 0.000 \\
\hline $\begin{array}{c}\text { Tamaño de } \\
\text { la camada } \\
\text { Peso al }\end{array}$ & \pm 2.4 & $9.7 \pm 1.9$ & 0.069 & \pm 2.3 & 0.000 & $9.8 \pm 2.0$ & 0.004 & $8.3 \pm 2.3$ & 0.000 & $10.5 \pm 2.1$ & 0.000 \\
\hline $\begin{array}{l}\text { nacimiento } \\
\text { Peso a los }\end{array}$ & $6.3 \pm 0.9$ & $6.2 \pm 0.6$ & 0.178 & $7.1 \pm 0.7$ & 0.000 & $5.9 \pm 0.7$ & 0.000 & $6.9 \pm 0.8$ & 0.000 & $5.7 \pm 0.5$ & 0.000 \\
\hline $\begin{array}{l}21 \text { días } \\
\text { Glocosuria }\end{array}$ & $\begin{array}{c}34.1 \pm 6.9 \\
\%\end{array}$ & $\begin{array}{c}32.6 \pm 5.3 \\
\%\end{array}$ & 0.032 & $\begin{array}{c}41.6 \pm 5.2 \\
\%\end{array}$ & 0.000 & $\begin{array}{c}32.1 \pm 5.4 \\
\%\end{array}$ & 0.000 & $\begin{array}{c}38.7 \pm 5.2 \\
\%\end{array}$ & 0.000 & $\begin{array}{c}28.4 \pm 4.9 \\
\%\end{array}$ & 0.000 \\
\hline Positiva & 62 & $42 \mathrm{O}$ & 0.077 & $54 \mathrm{O}$ & 0.002 & $42 \mathrm{O}$ & 0.035 & $28 \mathrm{O}$ & 0.040 & $22 \mathrm{O}$ & 0.001 \\
\hline Negativa & 38 & $58 \mathrm{~A}$ & 0.000 & $46 \mathrm{~A}$ & 0.59 & $\begin{array}{l}45 \mathrm{~A} \\
12 \mathrm{O} \\
1 \mathrm{~A}\end{array}$ & $\begin{array}{l}0.004 \\
0.007 \\
0.000\end{array}$ & $\begin{array}{ll}31 & \mathrm{~A} \\
21 & \mathrm{O} \\
20 & \mathrm{~A}\end{array}$ & $\begin{array}{l}0.168 \\
0.423 \\
0.000\end{array}$ & $\begin{array}{l}11 \mathrm{~A} \\
46 \mathrm{O} \\
21 \mathrm{~A}\end{array}$ & $\begin{array}{l}0.000 \\
0.000 \\
0.000\end{array}$ \\
\hline
\end{tabular}

Tabla 1: Configuración de los clusters a través de las variables activas (promedio \pm desvío estándar) y de la ilustrativa glucosuria (\% original: $O$ y asignado: $A$ ). Se incluye la probabilidad asociada al valor test que compara el valor de la clase con el general.

el propio modelo eSS (Tarrés et al. 1981, Tarrés et al. 1986, Martínez et al. 1988, Tarrés et al. 1990, Tarrés et al. 1992, Martínez et al 1993) y en la cepa recombinante producto del cruzamiento entre la línea b obesa (Calderari et al. 1995) y eSS, denominada $e S M T$ (Tarrés et al. 2000, Méndez et al. 2002).

En nuestro caso, la evolución del síndrome diabético que se da entre las clases 1 a 5 , se acompaña con un incremento de la edad en el mismo sentido, demostrando la correspondencia entre el envejecimiento y el aumento de las alteraciones del metabolismo glúcido de las ratas $e S S$.

Igualmente destacado resulta el aporte de la biomasa, ya que se ha señalado la importancia de dicha variable tanto en la diabetes humana (Diabetes and Nutrition Study Group of the European Association for the Study of Diabetes 1995) como en síndromes diabéticos de roedores (Ikeda et al. 1981, Koletsky y Ernsberger 1996). Aún cuando las ratas $e S S$ no son obesas, el mayor peso constituye un factor agravante de sus perturbaciones metabólicas (Tarrés et al. 1986, Martínez et al. 1988, Martínez et al. 1990, Martínez et al. 1993) y, en la tipificación aquí presentada, un aumento de la biomasa acompaña a las glucemias progresivamente mayores desde la clase 5 hasta la clase 2 . El menor peso registrado en la clase 1 podría deberse al deterioro producido por la enfermedad, dados los elevados valores de las variables metabólicas y la presencia de glucosuria. El descenso de peso luego de los 12 meses de edad ya había sido comprobado en esta línea coincidiendo con el agravamiento metabólico (Tarrés et al. 1981).

En el análisis efectuado en este trabajo, el segundo eje factorial opuso el tamaño de camada al peso al nacimiento y al destete lo que, en principio, indicaría muy escasa vinculación con el resto de las variables que conforman el primer eje factorial. En otras palabras, el tamaño de camada, el peso al nacimiento y al destete no siguen como las otras 
variables el "efecto tamaño".

La falta de una ubicación ordenada de estas variables en las clases construidas podría explicarse teniendo en cuenta los hallazgos de Kind et al. (2003) quienes comprobaron, en un estudio efectuado en guinea pig, que el retardo en el crecimiento fetal expresado por bajo peso al nacer se correlacionó, en la adultez, con hiperinsulinemia y resistencia a la insulina aunque sin alteración de la tolerancia a la glucosa, siendo precisamente las variables glucémicas las utilizadas en nuestra tipificación.

Por último, si se relaciona la clasificación de la Asociación Americana de Diabetes presentada más arriba con los clusters obtenidos a partir de los datos, puede resumirse entonces la tipología obtenida, de acuerdo con sus variables más características, de la siguiente manera:

CLASE 1: Formada por animales con edad, peso y glucemias superiores al promedio general, compatibles con diabetes en sus cuatro determinaciones y con el 100\% de glucosurias positivas.

CLASE 2: Constituida por individuos con edad, peso y glucemias superiores al promedio general aunque, a excepción de la biomasa, menores que en la CLASE 1, con G0 alterada y G30, G60 y G120 compatibles con diabetes y con el 100\% de glucosurias positivas. También pueden encontrarse aquí los que provienen de los menores tamaños de camada y tienen los mayores pesos al nacimiento y al destete.

CLASE 3: Integrada por ratas con edad, peso y glucemias superiores al promedio general, menores que en la CLASE 2, con G0 normal y G30, G60 y G120 compatibles con diabetes. Presentan, entre las observadas y las adjudicadas, el $87 \%$ de glucosurias positivas y el $13 \%$ de negativas

CLASE 4: Compuesta por sujetos con edad, peso y glucemias menores que en la clase anterior e inferiores al promedio general, con G0 normal y G30, G60 y G120 indicadoras de diabetes y en los que se registra, entre las observadas y las adjudicadas, el $58 \%$ de glucosurias positivas y el $42 \%$ de negativas.

CLASE 5: Conformada por animales con edad, peso y glucemias inferiores a los de la CLASE 4 y menores al promedio general, con G0 normal, G30 y G60 compatibles con diabetes y G120 indicadora de intolerancia a la glucosa. En esta clase se registra un $33 \%$ de glucosurias positivas y $67 \%$ de negativas, entre los valores observados y adjudicados. Se registran también los individuos con los tamaño de camada mayores y los menores pesos en edades tempranas.

Esta configuración espacial de los individuos permitió detectar diferencias fenotípicas multidimensionales, estructura que podría interpretarse como verdaderas fases (Hansen and Bodkin 1986, Mori et al. 1993) en el desarrollo de la diabetes de las ratas eSS, determinadas por la fuerte influencia de la edad dada su correspondencia con los ordenamientos descriptos, desde los mayores de 1 año en la clase 1 hasta los más jóvenes en la clase 5 .

Siendo la diabetes tipo 2 un síndrome no detectable al nacer por lo que se lo denomina, al igual que otros con la misma característica como la obesidad, "fenotipo tardío" 
(Williams 1984) y considerando, además, que es un desorden progresivo (Franz 2000), la identificación temprana de individuos en riesgo permitiría la aplicación oportuna de medidas preventivas (Lakka et al. 2002); téngase en cuenta que esta entidad se diagnostica en forma casual y tardía en casi el $70 \%$ de los casos, por lo que en ese momento ya suele presentar complicaciones crónicas (Gagliardino 2003).

Puede concluirse que la aplicación del análisis multidimensional posibilitó concretar una tipología de las ratas eSS a partir de un conjunto de variables metabólicas y de naturaleza ambiental, evaluando el aporte de estas últimas a la expresión del síndrome diabético y confirmando así la utilidad de los algoritmos clasificatorios en el abordaje de problemas biológicos.

\section{Referencias}

[1] Aluja, B.; Morineau, A. (1999) Aprender de los Datos: el Análisis de Componentes Principales. Ed. EUB, Barcelona, .

[2] Anderson, T.W. (1963) "Asymptotic theory for principal component analysis", Annals of Mathematic Statistics 34: 122.

[3] American Diabetes Association (2004) "Diagnosis and classification of diabetes mellitus", Diabetes Care 27 (Sup 1): 5.

[4] Buschard, K. (2001) "Meet a research group. Diabetes research group, Bartholin Instituttet, Copenhagen, Denmark",Scand. J. Lab. Sci. 28: 58.

[5] Calderari, S.; Font, M.T,; Garrocq, O.; Martínez, S.M.; Morini, J.C.; Puche, R.; Tarrés, M.C. (1991) "The inbred IIM/Fm stock", Rats News Letter 25: 28.

[6] Calderari, S.A.; Martínez, S.M.; Tarrés, M.C.; Picena, J.C.; Rabasa, S.L. (1995) "Modelos hereditarios de diabetes y obesidad en líneas endocriadas vinculadas por ascendencia", Mendeliana 11: 47.

[7] Carrasco, J.L.; Hernán, M.A. (1993) Estadística Multivariante en las Ciencias de la Vida. Fundamentos, Métodos y Aplicación. Ed Ciencia 3, SL. Madrid.

[8] Cattell, R.B. (1966) "The scree test for the number of factors", Multivariate Behavioral Research 1: 245.

[9] Costa, B.; Pinol, J.L.; Martin, F.; Donado, A.; Castell, C. (2002) "Significant incidence of type 2 diabetes on high-risk Spanish population", The IGT Study (2), Med. Clin. (Barc) 118: 287.

[10] Delemarre-van de Waal, H.A.; van Coeverden, S.C.; Engelbregt, M.T. (2002) "Factors affecting onset of puberty", Horm. Res. 57(Suppl. 2): 15.

[11] Diabetes and Nutrition Study Group of the European Association for the Study of Diabetes (1995) "Recomendations for the nutritional management of patients with diabetes mellitus", Diab. Nutr. Metab. 8: 186. 
[12] Dietterich, T.G. (2000) "Ensemble methods in machine learning", In Lecture Notes in Computer Science, First International Workshop on Multiple Classifier Systems. Kittler and F. Roll eds, Ed. Springer-Verlag 1.

[13] Diday, E. (1971) "La méthode des nuées dynamiques", Revue Statist. Appl. 19: 19.

[14] Duda, R.O.; Hart, P.E.; Stork, D.G. (2001) Pattern Classification Ed. John Wiley \& Sons, New York.

[15] Escofier, B.; Pagès, J. (1992) Análisis factoriales simples y múltiples. Universidad del País Vasco, Ed. Bilbao.

[16] Franz, M. (2000) "Does diet fail?", Clinical Diabetes.

[17] Gagliardino, J.J. (2000) "An overview of Argentine contributions to diabetes research in the decade of the 1990s", Diabetes Metab. Res. Rev. 16: 43.

[18] Gagliardino, J.J. (2003) "Epidemiología de la diabetes mellitus" (Simposio Diabetes, Buenos Aires), Medicina 63: 504.

[19] Hansen, B.C.; Bodkin, N.L. (1986) "Heterogeneity of insulin responses: phases leading to Type 2 (non-insulin-dependent) diabetes mellitus in the rhesus monkey", Diabetologia 29: 713 .

[20] Herdzik, E.; Safranow, K.; Ciechanowski, K. (2002) "Diagnostic value of capillary glucose, fructosamine and glycosylated haemoglobin in detecting diabetes and other abnormalities compared to oral glucose tolerance test. Acta Diabetologica 39: 15.

[21] Ikeda, H.; Shino, A.; Matsuo, T.; Iwatsuka, H.; Suzuoki, Z. (1981) "A new genetically obese- hyperglycemic rat (Wistar fatty)", Diabetes 30: 1045.

[22] Organización Mundial de la Salud (1985) "Diabetes mellitus", Serie de Informes Técnicos $\mathbf{7 2 7}$.

[23] Kind, K.L.; Clifton, P.M.; Grant, P.A.; Owens, P.C.; Sohlstrom, A.; Roberts, C.T.; Robinson, J.S.; Owens, J. A. (2003) "Effect of maternal feed restriction during pregnancy on glucose tolerance in the adult guinea pig", Am. J. Phisiol. Regul. Integr. Comp. Physiol. 284: 140.

[24] Kokko, J.P. (1986) "Overview of renal physiology", in: Fluids and electrolytes, Saunders WB Eds. Philadelphia. p 841.

[25] Koletsky, R.J.; Ernsberger, P. (1996) "Phenotypic characterization of a genetically obese and hypertensive rat strain: SHROB/Kol", Rat Genome 2: 10.

[26] Kuzuya, T.; Nakagawa, S.; Satoh, J.; Kanazawa, Y.; Iwamoto, T.; Kobayashi, M.; Nanjo, K.; Sasaki, A.; Seino, Y.; Ito, C.; Shima, K.; Nonaka, K.; Kadowaki, T. (2002) "Report of the Committee on the classification and diagnostic criteria of diabetes mellitus: Discussion", Diabetes Research and Clinical Practice 55: 65. 
[27] Lakka, H.M.; Laaksonen, D.E.; Lakka, T.A.; Niskanen, L.K.; Kumpusalo, E.; Tuomilehto, J.; Salonen, J.T. (2002) "The metabolic syndrome and total and cardiovascular disease mortality in middle-age men", JAMA 288: 2709.

[28] Lebart, L.; Morineau, A.; Piron, M. (1995) Statistique Exploratoire Multidimensionelle, Ed. Dunod. Paris.

[29] Martínez, S.M.; Tarrés, M.C.; Montenegro, S.M.; Milo, R.; Picena, J.C.; Figueroa, N.; Rabasa, S.L. (1988) "Spontaneous diabetes in eSS rats", Acta diabetol. lat. 25: 303.

[30] Martínez, S.M.; Tarrés, M.C.; Montenegro, S.M.; Revelant, G.; Figueroa, N.; Alonso, D.; Laudanno, O.M.; D'Ottavio, A.E. (1990) "Intermittent dietary restriction in eSS diabetic rats. Effects on metabolic control and skin morphology", Acta diabetol lat 27: 329 .

[31] Martínez, S.M.; Tarrés, M.C.; Picena, J.C.; Montenegro, S.M.; Gagliardino, J.J.; Gómez Dumm, C.L.; D'Ottavio, A.E.; Naves, A.; Rabasa, S.L. (1993) "eSS rat, an animal model for the study of spontaneous non-insulin-dependent diabetes", in: Lessons from animal diabetes IV. Shafrir E. (Ed.) Smith-Gordon, London, 75.

[32] Méndez, F.; Montenegro, S.; Figueroa, N.; Picena, J.C.; Martínez, S.; Tarrés, M.C. (2002) "Enfoque multivariado para individualizar la influencia de la edad en la diabetes genética de ratas eSMT", $5^{\circ}$ Congreso Latinoamericano de Sociedades de Estadística (CLATSE V), Universidad Nacional de Tres de Febrero, Buenos Aires.

[33] Mori, Y.; Yokoyama, J.; Nemoto, M.; Nishimura, M.; Ikeda, Y. (1993) "Expression of diabetes, its genetics and complications in WBN rats with endocrine-exocrine pancreatic lesion", in: Lessons from animal diabetes IV, E. Shafrir (Ed.) Smith-Gordon, London 91.

[34] Moscoloni, N. (1992) "Técnicas de clasificación en la caracterización de poblaciones: alumnos de la Facultad de Ciencias Bioquímicas y Farmacéuticas de la UNR", Revista IRICE 3-4: 147.

[35] Pereira, M.A.; Kriska, A.M.; Joswiak, M.L.; Dowse, G.K.; Collins, V.R.; Zimmet, P.Z.; Gareeboo, H.; Chitson, P.; Hemraj, F.; Purran, A. (1995) "Physical inactivity and glucose intolerance in the multiethnic island of Mauritius", Med. Sci. Sport Exerc. 27: 1626 .

[36] Picena, J.C.; Daniele, S.M.; Arriaga, S.; Martínez, S.M.; Tarrés, M.C.; Montenegro, S.; Hisano, N.; Morisoli, L.; D'Ottavio, A.E. (2002) "Analogías entre las ratas espontaneamente diabéticas eSS y pacientes diabéticos, con nefropatía y sin retinopatía", Medicina (Buenos Aires) 62: 508.

[37] Poulos, S.P.; Sisk, M.; Hausman, D.B.; Azain, M.J.; Hausman, G.J. (2001) "Pre- and postnatal dietary conjugated linoleic acid alters adipose development, body weight gain and body composition in Sprague-Dawley rats", J. Nutr. 131: 2722. 
[38] Ramos, R.R.; Flores S., J.L.; Téllez B., L.E.; Rivas V., J.F.; Alarcón A., F.J. (2000) "Estudio experimental de la acción antihiperglucemiante del nopal (Opuntia streptacantha Lemaire)", Animales de Experimentación (The Spanish-Language of Laboratory Animal Science \& Welfare) 5: 17.

[39] Roselló-Araya, M. (2003) "Prevalencia de diabetes tipo 2, intolerancia a la glucosa y diabetes provisional en El Guarco, Cartago", Rev. Costarric Cienc. Méd. 24: 15.

[40] Shafrir, E. (1996) "Development and consequences of insulin resistance: Lessons from animals with hyperinsulinemia", Diab. \& Metab. 22: 122.

[41] Solari, A.J. (1999) Genética Humana. Fundamentos y Aplicaciones en Medicina, $2^{a}$. Edición. Ed. Médica Panamericana S.A., Buenos Aires.

[42] SPAD version 4.02 (1999) "Logiciel d'Analyse des Données", CISIA-CERESTA.

[43] Strickberger M.W. (1988) "Efectos ambientales y expresión génica", in: Genética, Tercera Edición, Eds. Omega S.A., Barcelona.

[44] Supplement IV of the International Survey on the Supply, Quality and Use of Laboratory Animals (1964), On the Advise of the International Commitee on Laboratory Animals, NCR Labs, Carshalton, Surrey.

[45] Tarrés, M.C.; Martínez, S.M.; Liborio, M.M.; Picena, J.C.; Rabasa, S.L. (1986) "Efecto del ambiente nutricional sobre la expresión del síndrome diabético de las ratas eSS", Medicina (Buenos Aires) 46: 429.

[46] Tarrés, M.C.; Martínez, S.M.; Liborio, M.M.; Rabasa, S.L. (1981) "Diabetes mellitus en una línea endocriada de ratas", Mendeliana 5: 39.

[47] Tarrés, M.C.; Martínez, S.M.; Montenegro, S.M.; Picena, J.C.; Llorens, A.; Naves, A. (1992) "The eSS rat: a model of non- insulin- dependent human diabetes", Am. J. Pathol. 141: 761.

[48] Tarrés, M.C.; Martínez, S.M.; Montenegro, S.M.; Picena, J.C.; Naves, A.; Figueroa, N.S.; Rabasa, S.L. (1990) "Relación entre dieta, biomasa y expresión de la diabetes en la rata eSS", Medicina (Buenos Aires) 50: 235.

[49] Tarrés, M.C.; Montenegro, S.M.; Martínez, S.M.; Picena, J.C.; Toniolo, F.; Figueroa, N.S.; Naves, A. (2000) "The eSMT rat: a murine model of type 2 human diabetes", Proceedings of the International Joint Meeting XII ICLAS General Assembly \& Conference, VII FELASA Symposium. Chapter: Animals models in the study of human diseases, Laboratory Animals Ltd., London, UK. p 93.

[50] Traversa, M.; Ruiz, M. (1994) "Clasificación y diagnóstico", en: M. Ruiz (Ed.) Diabetes Mellitus, Capítulo 6. Librería AKADIA Editorial, Buenos Aires: 136-141. 
[51] Tsukui, S.; Kobayashi, I. (1995) "Effects of age and obesity on glycated haemoglobin and 1.5-anhydroglucitol in screening for type 2 diabetes mellitus", Diabet. Med. 12: 899.

[52] Uusitupa, M.I. (1996) "Early lifestyle intervention in patients with non-insulindependent diabetes mellitus and impaired glucose tolerance", Ann. Med. 28: 445.

[53] Van Zutphen, L. F.M. (1999) "Laboratory animal science: A view on the edge of the millenium", Animal Research and Welfare. A partnership. Proceedings of the International Joint Meeting XII ICLAS General Assembly \& Conference. VII FELASA Symposium, p 1. 